\title{
Observations of Solar Flare Doppler Shift Oscillations with the Bragg Crystal Spectrometer on Yohkoh
}

\author{
John T. Mariska \\ E. O. Hulburt Center for Space Research, Code 7673, Naval Research Laboratory, \\ Washington, DC 20375 \\ mariska@nrl. navy.mil
}

\begin{abstract}
Oscillations in solar coronal loops appear to be a common phenomenon. Transverse and longitudinal oscillations have been observed with both the Transition Region and Coronal Explorer and Extreme Ultraviolet Imaging Telescope imaging experiments. Damped Doppler shift oscillations have been observed in emission lines from ions formed at flare temperatures with the Solar Ultraviolet Measurements of Emitted Radiation Spectrometer. These observations provide valuable diagnostic information on coronal conditions and may help refine our understanding of coronal heating mechanisms. I have initiated a study of the time dependence of Doppler shifts measured during flares with the Bragg Crystal Spectrometer (BCS) on Yohkoh. This Letter reports the detection of oscillatory behavior in Doppler shifts measured as a function of time in the emission lines of S XV and Ca XIX. For some flares, both lines exhibit damped Doppler shift oscillations with amplitudes of a few $\mathrm{km} \mathrm{s}^{-1}$ and periods and decay times of a few minutes. The observations appear to be consistent with transverse oscillations. Because the BCS observed continuously for almost an entire solar cycle, it provides numerous flare data sets, which should permit an excellent characterization of the average properties of the oscillations.
\end{abstract}

Subject headings: Sun: flares — Sun: corona — Sun: oscillations — Sun: X-rays, gamma rays

\section{INTRODUCTION}

Sequences of images made with the Transition Region and Coronal Explorer (TRACE) show that a small subset of large-scale loops appear to respond to nearby flares by exhibiting 
damped transverse oscillations (e.g., Aschwanden et al. 1999; Schrijver et al. 2002). Damped Doppler shift oscillations have also been detected over active regions with the Solar Ultraviolet Measurements of Emitted Radiation (SUMER) experiment on the Solar and Heliospheric Observatory (SOHO) (e.g., Kliem, et al. 2002; Wang et al. 2003). These Doppler shifts are primarily observed in emission lines from ions formed at temperatures greater than $6 \mathrm{MK}$, and thus are probably associated with flare-like events.

The study of these oscillations is fast developing into a new technology for diagnosing the the physical conditions in the loops and, hence, placing further constraints on possible coronal heating mechanisms (e.g., Roberts 2002, 2003).

Detection of Doppler shifts in high-temperature emission lines with SUMER suggests that they might also be detectable with instruments capable of measuring Doppler shifts in soft X-ray lines, such as the Bragg Crystal Spectrometer (BCS) on the Yohkoh spacecraft. Seely \& Feldman (1984) have in fact detected some evidence for oscillations with a period of about 10 minutes in the position of the Ca XIX resonance line during the decay of a limb flare observed on 1980 November 13 with the SOLFLEX crystal spectrometer on the Pr8-1 satellite.

In this Letter I show that damped Doppler shift oscillations are present in some of the flares observed with the BCS.

\section{BCS OBSERVATIONS}

The Yohkoh BCS has been described in detail by Culhane et al. (1991). Four bent crystals cover narrow wavelength ranges centered on emission lines of Fe XXVI, Fe XXV, Ca XIX, and S XV. Radiation diffracted by each crystal strikes a one-dimensional positionsensitive proportional counter, with the Fe XXVI and Fe XXV crystals sharing one detector and the Ca XIX and S XV crystals sharing a second detector. All four detectors view the entire Sun. For this study I use data from the Ca XIX and S XV channels. Examples of the appearance of the spectra in these two channels are available in, for example, Doschek et al. (1992).

To search for Doppler shift oscillations in the BCS data, I have initiated an examination of the temporal behavior of the physical characteristics of a large number of the flares observed by the BCS over its 10-year lifetime. Fitting the BCS data using synthetic spectra yields estimates of the temperature, emission measure, nonthermal broadening, and Doppler shift of the emitting plasma as a function of time. The best fit parameters are determined by computing synthetic spectra for an isothermal plasma and then varying the 
fitting parameters to minimize the $\chi^{2}$ statistic.

In most cases, the BCS obtains spectra in all four wavelength channels every $3 \mathrm{~s}$. The Fe XXVI channel rarely contains useful data, and obtaining Doppler shifts from the Fe XXV data is made difficult by the complexity of the spectra in that channel. Thus, this study is restricted to data from the S XV and Ca XIX. Early or late in a flare, the instrument count rates are small, resulting in spectra that are difficult to fit. For this survey, I have therefore summed individual spectra until a minimum of 10,000 counts are accumulated in the S XV and Ca XIX channels. This generally results in excellent fits to the data and assures that each data point in the resulting time series of physical parameters will have roughly the same statistical significance. It does, however, mean that the data points in the two derived time series for each flare will not generally be at identical times.

I have analysed data for more than 100 flares observed by the BCS. Most of the data examined to date cover the first year of the mission. In addition I have examined flares listed in Schrijver et al. (2002) for which there are useful BCS data. A significant number of the flares observed (roughly 25\%) show evidence for oscillatory behavior. In the following section, I show the results for one event that exhibited evidence of damped Doppler shift oscillations.

\section{RESULTS}

Figure 1 shows the temporal behavior of the count rates and the physical parameters resulting from fits to the individual spectra for the S XV and Ca XIX wavelength channels in one flare that exhibits damped oscillatory behavior. This C2.5 flare took place at S16W88 on 1992 October 2, and is one of the nonocculted limb flares in the survey of occulted and nonocculted limb flares performed by Mariska \& McTiernan (1999). Nonocculted flares in that study refers to events for which images obtained with the Yohkoh Soft X-Ray Telescope (SXT) showed that the footpoints of the flaring loops were not occulted by the solar limb. At the peak of the flare, the region of strong emission in the SXT images occupies only a few pixels above the solar limb, suggesting that the hot plasma detected with the BCS is confined to a relatively small volume.

While the total count rate, temperature, emission measure, and nonthermal velocity show generally smooth behavior as a function of time, the Doppler shift shows evidence of oscillatory behavior in both channels. In particular, the Doppler shift in the S XV channel appears to show evidence for a damped oscillation.

To determine the characteristics of the oscillations observed in Figure 1, I follow the 
example of earlier work on TRACE oscillations (e.g., Aschwanden et al. 2002) and fit the observations with a combination of a damped sine wave and a polynomial background. For each channel I define a function of the form

$$
v(t)=A_{0} \sin (\omega t+\phi) \exp (-\lambda t)+B(t),
$$

where

$$
B(t)=b_{0}+b_{1} t+b_{2} t^{2}+b_{3} t^{3}+\cdots
$$

is the trend in the background data. Starting from an initial guess for the fit parameters, the data in each channel were fit to Equation (1) using Levenberg-Marquardt least-squares minimization (e.g., Press et al. 1992). For all the flares studied the number of terms needed for the background expression was generally one or two, although occasionally three terms produced a better fit.

The Doppler shift data shown in Figure 1 are sufficiently noisy that it is difficult to fit them with Equation (1). Since it is clear from the figure that the period of the sine wave is on the order of a few minutes, I have accumulated the BCS data for longer intervals by requiring that the minimum number of counts in a channel exceed 10,000 and that the accumulation time be at least $20 \mathrm{~s}$. The top panels in Figure 2 show the Doppler shift data for the two channels accumulated in this manner. Also plotted on the top panels is the best fit background trend. For the S XV spectra, a two-term expression provided the best fit, while for Ca XIX, one term was sufficient.

The bottom two panels of Figure 2 show the background-subtracted data along with the best-fit damped sine wave. For both channels, only the regions between the vertical dashed lines were used in the fitting process. Even with the longer accumulation time the data are still noisy, especially those from the Ca XIX channel. The fits to the data, however, do appear to be reasonable. Reduced $\chi^{2}$ values are 5.8 and 1.2 for the S XV and Ca XIX channels, respectively.

Table 1 summarizes the fitting results. While the origin of time for the two channels was tied to the first fitted spectrum, the values in the table for Ca XIX have been adjusted to correspond to the same initial time used for the S XV fits. The errors listed in the table correspond to the diagonal elements in the covariance matrix for the fits. Examination of the results in the table shows that both BCS channels show evidence for the same oscillatory behavior in the Doppler shift. This was generally the case with the other flares that showed damped oscillatory behavior. 


\section{DISCUSSION AND CONCLUSIONS}

Figure 1 shows that for the first several minutes of the flare the BCS S XV and Ca XIX channels show different plasma temperatures. The comparable oscillation parameters in Table 1 thus indicate that the flaring structures at the two temperatures are closely related. This suggests that the oscillations are not restricted to just one isolated loop.

The measured values for the periods (3.93 and $3.57 \mathrm{~min})$ and decay times (3.52 and $4.26 \mathrm{~min}$ ) fall near the low side of the range of values listed for the 26 oscillating loops

observed with TRACE by Aschwanden et al. (2002). Following Wang et al. (2003), we define the maximum displacement amplitude for each fit as

$$
A=A_{0} /\left(\omega^{2}+\lambda^{2}\right)^{1 / 2} .
$$

This yields displacements of 416 and $295 \mathrm{~km}$ for S XV and Ca XIX, respectively. These values are less than 1 arcsec. Thus, if the oscillations are due to a position change in the magnetic structure confining the flaring plasma, the change would be invisible at the roughly 3 arcsec spatial resolution of the SXT. This inferred excursion also falls near the low side of the range listed by Aschwanden et al. (2002). All the above values fall below the ranges listed by Wang et al. (2003) for Doppler shift oscillations observed with SUMER.

The measured values for the oscillations shown in the the table and figures are typical of those determined for the other flares with well-defined oscillations. Those flares tend to be at or near the solar limb, which, since BCS measures only line-of-sight Doppler shifts is where one would expect to most clearly see oscillatory behavior. Many other flares in the sample show evidence for significant Doppler shift fluctuations, but the data are not easily fitted with a model such as that used in Equation (1). This would suggest that there are many cases where different structures in the flaring plasma are moving in an uncoordinated fashion, leading to a complex signal in the BCS, which observes all the flaring plasma.

Since the BCS has no imaging capability and integrates over all the plasma contributing photons to the emission observed in each wavelength channel, it is not possible to determine from the Doppler shift data alone whether we are observing transverse or longitudinal oscillations. The periods and decay times measured for the event shown in this Letter and those measured for other events in the study are more typical of those seen in the TRACE observations (Aschwanden et al. 2002) - suggesting that the BCS observations are detecting transverse oscillations. On the other hand, the ratio of the decay time to the period is roughly 0.9 , more typical of the results obtained by Wang et al. (2003).

Unfortunately, none of the flare-associated events listed in (Wang et al. 2003) had useful BCS data. A number of the events analyzed by (Aschwanden et al. 2002) have useful BCS 
observations, and those events are included in a more comprehensive analysis of the BCS Doppler shift observations, which is currently in progress (Mariska 2005). The results of that analysis should provide a firmer estimate of the range of oscillation properties seen in the BCS data and help determine whether they are transverse or longitudinal oscillations.

I thank G. A. Doschek, H. Hudson, and H. P. Warren for their comments on the manuscript. This research was supported by ONR/NRL 6.1 basic research funds.

\section{REFERENCES}

Aschwanden, M. J., DePontieu, B., Schrijver, C. J., \& Title, A. M. 2002, Sol. Phys., 206, 99

Aschwanden, M. J., Fletcher, L., Schrijver, C. J., \& Alexander, D. 1999, ApJ, 520, 880

Culhane, J. L. et al. 1991, Sol. Phys., 136, 89

Doschek, G. A. et al. 1992, PASJ, 44, L95.

Kliem, B., Dammasch, I. E., Curdt, W., \& Wilhelm, K. 2002, ApJ, 568, L61

Mariska, J. T. 2005, ApJ, in preparation

Mariska, J. T., \& McTiernan, J. M. 1999, ApJ, 514, 484

Press, W. H., Teukolsky, S. A., Vetterling, W. T., \& Flannery, B. P. Numerical Recipes in C (2d ed.; Cambridge: Cambridge Univ. Press)

Roberts, B. 2002, Sol. Phys., 193, 139

Roberts, B. 2003, in SOHO13, Waves and Small Scale Transient Events in the Solar Atmosphere: A Joint View from SOHO and TRACE, ESA SP-547

Schrijver, C. J., Aschwanden, M. J, \& Title, A. M. 2002, Sol. Phys., 206, 69

Seely, J. F., \& Feldman, U. 1984, ApJ, 280, L59

Wang, T. J., Solanki, S. K., Curdt, W., Innes, D. E., Dammasch, I. E., \& Kliem, B. 2003, A\&A, 406, 1105 
Table 1. Fitting Results

\begin{tabular}{lcc}
\hline \hline \multicolumn{1}{c}{ Parameter } & S XV & Ca XIX \\
\hline$A_{0}\left(\mathrm{~km} \mathrm{~s}^{-1}\right)$ & $11.3 \pm 1.9$ & $8.76 \pm 2.78$ \\
$\omega(\mathrm{rad} \mathrm{min}$ & -1 \\
$\phi(\mathrm{rad})$ & $1.60 \pm 0.06$ & $1.76 \pm 0.17$ \\
$\lambda\left(\mathrm{min}^{-1}\right)$ & $-2.43 \pm 0.05$ & $-2.86 \pm 0.41$ \\
\hline
\end{tabular}



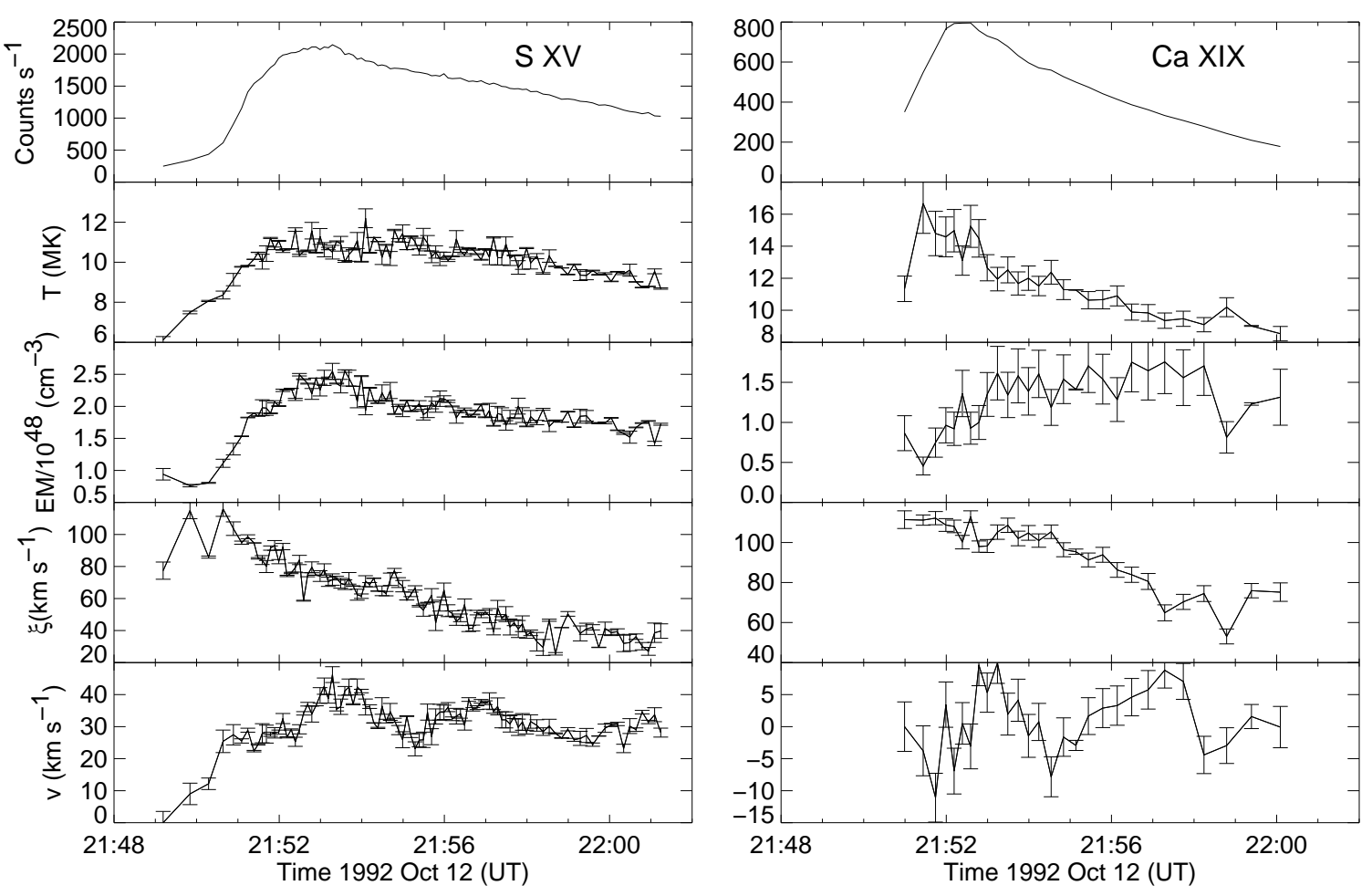

Fig. 1.- Temporal behavior of the temperature, emission measure, nonthermal broadening velocity, and Doppler shift derived from the BCS S XV and Ca XIX observations for the 1992 October 12 flare. The zero value for the Doppler shift velocities has been set to the wavelength shift of the first fitted spectrum. 

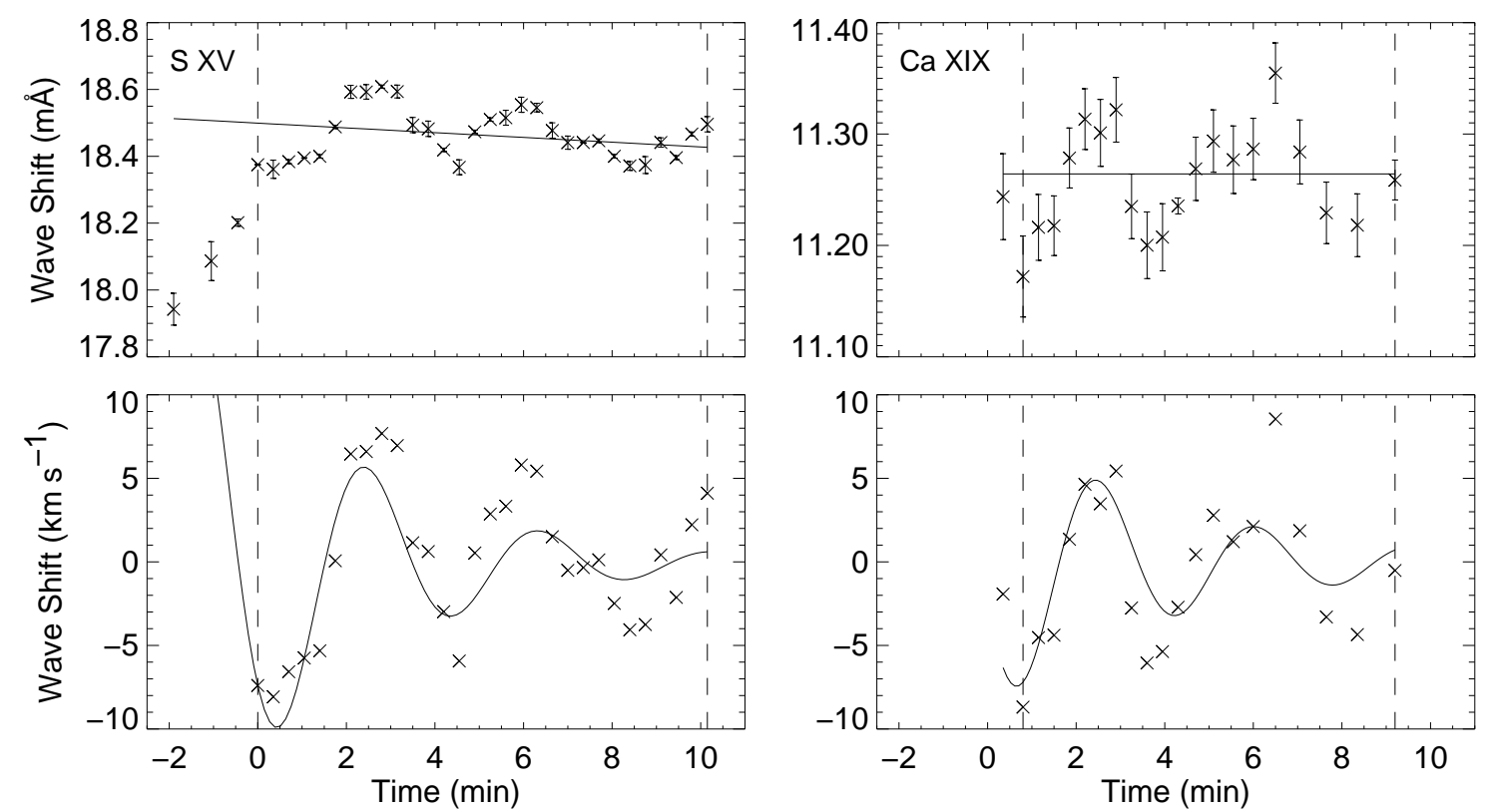

Fig. 2.- Averaged BCS Doppler shift data and decaying sin wave fits for the S XV and Ca XIX observations for the 1992 October 12 flare. The zero value for the shifts plotted in the top panels is based on an arbitrary reference system for the BCS in which a flare near the solar Equator with no Doppler shift would have a value of $0 \mathrm{~m} \AA$. Time is measured from 21:50:44 UT. 